Trauma Surgery \& Acute Care Open

\title{
Marginal ulcer with erosion into splenic artery in a patient with a Roux-en-Y gastric bypass
}

\author{
Jennifer Silvis, ${ }^{1}$ Leah Aakjar, ${ }^{1}$ Jane Keating, ${ }^{1,2}$ Nicholas Costanzo, ${ }^{1,2}$ Pallavi Nadendla, ${ }^{3}$ \\ Daniel Ricaurte (1) 1,2
}

'University of Connecticut School of Medicine, Farmington, Connecticut, USA ${ }^{2}$ Hartford Hospital, Hartford, Connecticut, USA ${ }^{3}$ Interventional Radiology, Hartford Hospital, Hartford, Connecticut, USA

Correspondence to Dr Daniel Ricaurte; daniel. ricaurte@hhchealth.org (c) Author(s) (or their employer(s)) 2021. Re-use permitted under CC BY-NC. No commercial re-use. See rights and permissions. Published by BMJ.

\section{To cite:}

Silvis J, Aakjar L, Keating J,

et al. Trauma Surg Acute Care

Open 2021:6:e000784.
A female patient in her 70s with a remote history of an open Roux-en-Y gastric bypass for weight loss was admitted to the medical intensive care unit (ICU) for septic arthritis of the hip and associated bacteremia. On hospital day 7 , the patient was found to have a significant drop in hemoglobin to $55 \mathrm{~g} / \mathrm{L}$. Despite undergoing a CT angiography of the abdomen and pelvis, no source of bleeding was identified. She received two units of blood and responded appropriately. On hospital day 9, the patient 4 began to experience melanic stools and hematemesis. At that time, she was found to have a hemoglobin of $40 \mathrm{~g} / \mathrm{L}$, a base deficit of -15 , and was requiring three vasopressors to maintain adequate blood pressure. Both acute care surgery as well as gastroenterology were emergently consulted. A femoral venous introducer was placed and a massive transfusion protocol (MTP) was initiated. The patient was intubated, and an ultrasoundguided femoral arterial line was placed. The patient responded appropriately to MTP, calcium repletion, and was able to wean off vasopressors, prompting the cessation of the MTP. Emergent bedside upper endoscopy performed by gastroenterology revealed a marginal ulcer at the gastrojejunostomy with a visible vessel. This was injected with epinephrine and clipped. Despite these attempts, the bleeding was persistent and unable to be controlled endoscopically. At that time, the patient was not requiring vasopressors or ongoing transfusions to maintain appropriate blood pressure.

\section{WHAT WOULD YOU DO?}

A. Proceed to the operating room for an exploratory laparotomy?

B. Proceed to the operating room for exploratory laparoscopy?

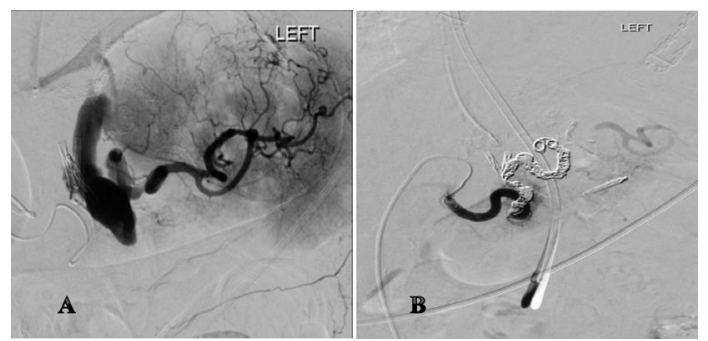

Figure 1 (A) Mesenteric angiography showing pseudoaneurysm with contrast extravasation from the splenic artery. (B) Completion angiogram after Gelfoam embolization showing no further extravasation of contrast.
C. Proceed to interventional radiology for mesenteric angiogram?

\section{WHAT WE DID AND WHY}

\section{Correct answer $\mathrm{C}$}

In light of the patient's hemodynamic stability at the conclusion of the upper endoscopy, the decision was made to proceed to interventional radiology for mesenteric angiography. The femoral arterial access was upsized for a 7 French sheath and a REBOA catheter was kept at the bedside in the event the patient became hypotensive in transport to radiology. During the mesenteric angiogram, a large pseudoaneurysm with contrast extravasation was found arising from the splenic artery, successfully treated with coil embolization. Completion angiogram demonstrated no further extravasation with reconstitution of the distal splenic artery via the pancreaticoduodenal arcade (figure 1).

The patient was transferred to the surgical ICU for ongoing resuscitation. On post-procedure day 4 , the patient experienced another episode of melena requiring transfusion. Repeat mesenteric angiography demonstrated a hypertrophied left gastric artery, which was embolized with Gelfoam. On post-procedure day 7 , the patient underwent repeat upper endoscopy for a slow drift in hemoglobin which did not show any active bleeding.

Marginal ulcers are a common complication of gastric bypass. Risk factors for marginal ulcers include smoking, diabetes, and non-steroidal antiinflammatory drug use. The most common presentations for marginal ulcers include abdominal pain, nausea, and emesis. Rarely, patients may present in hemorrhagic shock. In these cases, the most common etiology is bleeding from jejunal branches of the superior mesenteric artery or gastric branches of the celiac trunk. These patients may present with a hostile abdomen especially if their index operation was performed in an open fashion. Only a handful of case reports have been published describing bleeding marginal ulcers from the splenic artery. To our knowledge, they have all been treated surgically with mixed success.

Massive hemorrhage from marginal ulcers is an emergency. First line of treatment should include endoscopic evaluation and early surgical consultation. If a marginal ulcer is the culprit and endoscopic therapies are unsuccessful in controlling the bleeding, consideration may be given to angiographic evaluation. This requires a hemodynamically appropriate patient. This clinical scenario 
intuitively lends itself to the use of REBOA and warrants further investigation.

Twitter Jane Keating @DrJaneKeating and Daniel Ricaurte @ricaurte_daniel

Contributors Authors JS and DR contributed to manuscript creation and revision. $L A$, JK, NC and PN contributed to manuscript revision.

Funding The authors have not declared a specific grant for this research from any funding agency in the public, commercial or not-for-profit sectors.

Competing interests None declared.
Patient consent for publication Not required.

Provenance and peer review Not commissioned; internally peer reviewed.

Open access This is an open access article distributed in accordance with the Creative Commons Attribution Non Commercial (CC BY-NC 4.0) license, which permits others to distribute, remix, adapt, build upon this work non-commercially, and license their derivative works on different terms, provided the original work is properly cited, appropriate credit is given, any changes made indicated, and the use is non-commercial. See: http://creativecommons.org/licenses/by-nc/4.0/.

\section{RCID id}

Daniel Ricaurte http://orcid.org/0000-0003-0284-4832 